\title{
Multi-objective Design of Induction Motor using Harmony Search Optimization
}

\author{
P.S. Prakash \\ Assistant Professor \\ Electrical Engineering, Annamalai University \\ India
}

\author{
P. Aravindhababu \\ Professor \\ Electrical Engineering, Annamalai University \\ India
}

\begin{abstract}
This paper presents a Harmony Search Optimization (HSO) based design methodology for maximizing both the starting torque and the efficiency of Induction Motor (IM). HSO is inspired the musical process of searching for a perfect state of harmony. The harmony in music is analogous to the optimization solution vector, and the musician's improvisations are analogous to local and global search schemes in optimization techniques. Among the number of design variables of the IM, seven variables are identified as primary design variables and the HSO based design methodology is tailored to optimize the chosen primary variables with a view to obtain the global best design. The optimal design obtained by the developed methodology for two IMs are presented with a view of illustrating the superiority.
\end{abstract}

\section{General Terms}

Optimization, evolutionary algorithms

\section{Keywords}

Harmony search optimization, Induction motor design

$\begin{array}{ll}\text { Nomenclature } \\ \text { BW } & \text { bandwidth } \\ E f f & \text { efficiency of the motor } \\ f(x) & \text { objective function to be minimized } \\ F I T & \text { fitness function } \\ g(x) & \text { a set of inequality constraints } \\ \text { HM } & \text { harmony memory } \\ \text { HMCR } & \text { harmony memory considering rate } \\ \text { HMS } & \text { harmony memory size } \\ \text { HSO } & \text { harmony search optimization } \\ h^{j} & j \text {-th harmony } \\ h_{i}^{j} & i \text {-th design variable of } j \text {-th harmony in the } \\ h^{\prime} & \text { HM } \\ \text { IM } & \text { improvised harmony vector } \\ I_{p h} & \text { induction motor } \\ I_{r} & \text { phase current, A } \\ & \text { equivalent rotor current, A }\end{array}$

\begin{tabular}{|c|c|}
\hline$I_{s c}$ & short circuit current per phase, $\mathrm{A}$ \\
\hline Iter ${ }^{\max }$ & $\begin{array}{l}\text { maximum number of iterations for } \\
\text { convergence check }\end{array}$ \\
\hline$k W$ & rating of IM \\
\hline $\begin{array}{l}\text { "min" \& } \\
\text { "max" }\end{array}$ & $\begin{array}{l}\text { minimum and maximum limits of the } \\
\text { respective variables }\end{array}$ \\
\hline$n d$ & number of decision variables \\
\hline ODIM & optimal design of IM \\
\hline PAR & pitch adjusting rate \\
\hline PSO & particle swarm optimization \\
\hline PM & proposed method \\
\hline$P_{t}$ & total losses \\
\hline$P_{n l}$ & no load loss \\
\hline$P_{c u s}$ & stator copper loss. \\
\hline$P_{c u r}$ & rotor copper loss. \\
\hline rand & a uniform random number between 0 and 1 \\
\hline$X$ & a vector of primary design variables \\
\hline$S F L$ & slip at full load, per unit \\
\hline$T_{s t}$ & starting torque, per unit \\
\hline $\mathfrak{R}_{i}$ & $\begin{array}{l}\text { a set possible range of values of } i \text {-th } \\
\text { decision variable, that is } \\
h_{i}(\min ) \leq \mathfrak{R}_{i} \leq h_{i}(\max )\end{array}$ \\
\hline$\eta$ & a set of limit violated constraints \\
\hline$w$ & $\begin{array}{l}\text { weight values to represent relative } \\
\text { significance }\end{array}$ \\
\hline$\lambda$ & weight constant of the penalty terms \\
\hline
\end{tabular}

\section{INTRODUCTION}

Although squirrel-cage induction machines are widely used in the industry for their easy manufacturing and robustness, their applications in electrical transport systems such as subways and trains are limited due to inferior starting torque than those of permanent-magnet motors. In the light of the fact that the resistance of a squirrel-cage motor is fixed and small as compared to its reactance that is very large especially at the start, the frequency of the rotor currents equals the supply 
frequency, the starting current of the rotor is very large in magnitude and lags by a very large angle, resulting a poor starting torque per ampere. It is roughly 1.5 times the full-load torque, although the starting current is 5 to 7 times the full load current. Hence, such motors are not useful where the motor has to start against heavy loads. Therefore it becomes imperative that the best architecture and the corresponding dimensioning have to be determined in order to maximize the starting torque besides improving the efficiency with respect to several constraints in applications requiring high starting torque such as in traction systems,. The resulting mathematical optimization problem is a multiobjective optimization problems and usually difficult since the design variables contain continuous variables related to the real dimensioning parameters and combinatorial variables associated with architecture characteristics and discrete dimensioning parameters; and their relationship with motor specifications are in general nonlinear [1].

In recent decades, several classical techniques such as nonlinear programming [2], Lagrangian relaxation method [3], direct and indirect search methods [4], Hooks and Jeeves method [5], Rosenbrock's method [6], Powell's method [7], finite element method [8] and sequential unconstrained minimization technique [9] have been suggested for IM design problem. Many of these methods are most cumbersome and time consuming and pose difficulty in handling non-linear and discontinuous objectives and constraints. Besides a few of them requires derivatives and exhibits poor convergence properties due to approximations in derivative calculations; and may converge to local solution instead of global ones, when the initial guess is in the neighborhood of a local solution.

In recent years nature inspired metaheuristic optimization algorithms such as simulated annealing [10], genetic algorithm (GA) [11], evolutionary algorithm [12], evolutionary strategy [13], particle swarm optimization (PSO) [14], bacterial foraging [15] and differential evolution [16] have been widely applied in solving the IM design problems with a view of overcoming the drawbacks of classical methods. These algorithms have yielded satisfactory results across a great variety of design optimization problems.

Recently, a Harmony Search Optimization (HSO) that was conceptualized using musical process of searching for a perfect state of harmony has been suggested for solving optimization problems [17,18]. The harmony in music is analogous to the optimization solution vector, and the musician's improvisations are analogous to local and global search schemes in optimization techniques. The HSO does not require initial values for the decision variables and uses a stochastic random search that is based on the harmony memory considering rate (HMCR) and pitch adjusting rate (PAR) so that the derivative information is unnecessary. It requires fewer mathematical computations compared to other meta-heuristic algorithms and can be easily adopted for various types of engineering optimization problems. It has been successfully applied in solving various engineering optimization problem in [19-21].

The aim of this paper is to develop a design methodology using HSO for maximizing the efficiency and starting torque of IM with a view of effectively exploring the solution space and obtaining the global best solution. The developed design methodology has been applied in designing two IMs and the performances have been studied. The paper is divided into five sections. Section 1 presents the introduction, section 2 overviews HSO, section 3 explains the IM design problem and suggests the proposed design method (PM), section 4 discusses the results and section 5 concludes.

\section{HARMONY SEARCH ALGORITHM}

The HSO is based on the musical process of searching for the perfect state of harmony. Musicians, during a rehearsal or a performance, try to create pleasing sounds and approach the ideal state of harmony. HSO is inspired from the improvisation process of music players. Just as the musicians try to improve their music, the HSO seeks for certain values for the decision variables that optimize the objective function while at the same time satisfying the problem constraints. It improves the optimal solution iteration after iteration in the same way as a music band improves rehearsal after rehearsal. It is simple in concept, few in parameters and easy for implementation with theoretical background of stochastic derivative.

In this approach, a Harmony Memory (HM), comprising a number of candidate solutions of the problem at hand, is defined. The HM is initialized with random guesses in the problem space as:

$$
H M=\left[\begin{array}{cccc}
h_{1}^{1} & h_{2}^{1} & \cdots & h_{N}^{1} \\
h_{1}^{2} & h_{2}^{2} & \cdots & h_{N}^{2} \\
h_{1}^{3} & h_{2}^{3} & \cdots & h_{N}^{3} \\
\vdots & \vdots & & \vdots \\
h_{1}^{H M S} & h_{2}^{H M S} & \cdots & h_{N}^{H M S}
\end{array}\right]
$$

Generating a new harmony is known as "improvisation". A new harmony vector $h^{\prime}=\left(h_{1}^{\prime}, h_{2}^{\prime}, \cdots, h_{N}^{\prime}\right)$ is generated based on the following mechanisms.

Memory considerations: The value for the first decision variable $h_{1}^{\prime}$ for the new vector is chosen from any of the values in the specified HM range $\left(h_{1}^{\prime}-h_{1}^{H M S}\right)$. Values for other decision variables $\left(h_{2}^{\prime}, h_{3}^{\prime}, \cdots, h_{N}^{\prime}\right)$ are chosen in the same manner. The HMCR that varies between 0 and 1 is the rate of choosing a value from HM, while (1-HMCR) is the rate of randomly selecting a value from the possible range of values as shown in (2)

$$
\begin{array}{ll}
\hline \text { if }(\text { rand }()<\mathrm{HMCR}) \\
& h_{i}^{\prime} \leftarrow h_{i}^{\prime}=\left(h_{i}^{1}, h_{i}^{2}, \cdots, h_{i}^{H M S}\right) \\
\text { else } & \\
& h_{i}^{\prime} \leftarrow h_{i}^{\prime} \in \Re_{i} \\
\text { end } & \\
\hline
\end{array}
$$

Pitch adjustment: Every component obtained by memory consideration is pitch adjusted based on the PAR as

$$
\begin{array}{ll|}
\hline \text { if }(\operatorname{rand}()<\text { PAR }) \\
\text { else } & h_{i}^{\prime} \leftarrow h_{i}^{\prime} \pm \operatorname{rand}() \times B W \\
& h_{i}^{\prime} \leftarrow h_{i}^{\prime} \\
\text { end } &
\end{array}
$$

Where BW is an arbitrary distance bandwidth.

Update harmony memory: If the new harmony vector $h^{\prime}=\left(h_{1}^{\prime}, h_{2}^{\prime}, \cdots, h_{N}^{\prime}\right)$ is better than the worst harmony in the $\mathrm{HM}$, then the worst harmony is replaced by the new harmony. 
Convergence Check: The process of generating new harmony vector can be terminated when the number of iterations reaches the maximum number of iterations.

\section{PROPOSED METHOD}

The proposed HSO based design method (PM) for ODIM involves formulation of the problem, representation of harmonies through the chosen design variables and construction of a fitness function, FIT .

\subsection{Problem Formulation}

The ODIM problem involves large number of design variables. Many of these variables fortunately have a little influence either on the objective function or on the specified constraints. However, to ease the curse of high dimensionality, the following seven variables are identified as primary design variables $(9 / 1)$.

$$
X=\left[x_{1}, x_{2}, \cdots, x_{7}\right]=\left[\begin{array}{l}
\text { Core length to polepitch } \\
\text { Average valueof air gap flux density } \\
\text { Ampere conductor } \\
\text { Lengthof air gap } \\
\text { Stator current density } \\
\text { Rotor current density } \\
\text { Fluxdensityin thecore }
\end{array}\right]^{T}
$$

The ODIM problem is formulated by defining an objective function through blending both the objectives with suitable weight values and a set of constraints as

Maximize $f(x)=w E f f+(1-w) T_{s t}$

Subject to

$g(x) \leq 0 \Leftrightarrow\left\{\begin{array}{l}\text { maximum flux densityof stator te๕ } \mathrm{h} \leq 2 \\ \text { maximum flux densityof rotor teeh } \leq 2.0 \\ \text { slipat full load } \leq 0.05 \\ \text { startingto full loadtorqueratio } \geq 1.5 \\ \text { stator temperaturerise } \leq 70 \\ \text { per unitnoloadcurrent } \leq 0.5 \\ \text { powerfactor } \geq 0.75\end{array}\right\}$

$$
x_{i}^{\min } \leq x_{i} \leq x_{i}^{\max } \quad i=1,2, \cdots n d
$$

Where

$$
\begin{aligned}
& E f f=\frac{K W}{K W+P_{t}} \\
& T_{s t}=\left(\frac{I_{s c}}{I_{r}}\right)^{2} * S F L \\
& I_{r}=0.85^{*} I_{p h} \\
& I_{s c}=\frac{V_{p h}}{Z} \\
& P_{t}=P_{n l}+P_{c u s}+P_{c u r} .
\end{aligned}
$$

\subsection{Representation of Design Variables}

The harmony, $h$ is represented to denote the chosen primary design variables, defined by Eq. (4), in vector form as:

$$
h^{i}=\left[h_{1}^{i}, h_{2}^{i} \cdots, h_{7}^{i}\right]=\left[x_{1}, x_{2}, \cdots, x_{7}\right]
$$

\subsection{Fitness Function}

The algorithm searches for optimal solution by maximizing a fitness function FIT, which is formulated from the objective function of Eq. (5) and the penalty terms representing the limit violation of the explicit constraints of Eq. (6). The fitness function is written as

$$
\text { Maximize } \quad F I T=\frac{f(x)}{1+\lambda \sum_{i \in \eta}\left[g_{i}(x)\right]^{2}}
$$

\subsection{Solution Process}

The process of generating a new harmony from the HM, which is generated randomly through memory considerations, pitch adjustment and memory update, may be called an iteration. The iterations are continued till the number of iterations reaches the a specified maximum number of iterations. The algorithmic steps of the PM are summarized below:

1. Read the IM data

2. Choose HSO parameters such as $H M S, H M C R$, $P A R, B W$ and Iter $^{\max }$

3. Randomly generate initial $H M$ consisting as many randomly generated solution vectors as the $H M S$

4. Perform the following for each harmony in the $H M$

- Obtain the primary design variables from the harmony.

- Compute the remaining secondary variables of the design problem.

- Evaluate FIT using Eq.(14)

5. Rank the $H M$ based on the fitness function FIT values

6. Set iteration counter $t=0$

7. Increment the iteration counter $t=t+1$

8. Evaluate $P A R(t)$ and $B W(t)$

9. Improvise a new harmony vector $x^{\prime}$ based on the probability $H M C R$

10. Adjust the pitch of each element of the new harmony based on the probability $P A R(t)$

11. Perform the following for new harmony

- Obtain the primary design variables from the new harmony.

- Compute the remaining secondary variables of the design problem.

- Evaluate FIT using Eq.(14)

12. Update the $H M$ by the new harmony, if the new harmony is better than the worst harmony of the $H M$

13. Repeat steps $7-12$ till the number of iterations, $t$, reaches the maximum number of iterations, Iter ${ }^{\max }$ 
14. The best harmony in the $H M$ is the optimal solution.

15. Stop.

\section{NUMERICAL RESULTS}

The proposed HSO based method is used to obtain the optimal design of two IMs. The first motor under study is rated for 7.5 $\mathrm{kW}, 400 \mathrm{~V}, 4$ pole, $50 \mathrm{~Hz}$ and the second one for $30 \mathrm{~kW}, 400$ $\mathrm{V}, 6$ pole, $50 \mathrm{~Hz}$. The effectiveness of the PM is illustrated through comparing the performances with that of the GA based design approach. In this regard, the same set of primary design variables, fitness function and design equations, involved in the PM, are used to develop the GA based design approach. The software packages are developed in Matlab platform and executed in a $2.67 \mathrm{GHz}$ Intel core-i5 personal computer. There is no guarantee that different executions of the developed design programs converge to the same design due to the stochastic nature of the GA and HSO and hence the algorithms are run 20 times for each IM and the best ones are presented.

Initially the designs are obtained by optimizing the individual objectives of efficiency and starting torque by setting the $w$ values as 1 and 0 respectively, and presented in Table 1 for both the motors. It is clear from the table that the PM is able to obtain the better efficiency when $w=1$ and better starting torque when $w=0$ than that of the GA approach. However, it is to be noted that the other performance value of efficiency for the case with $w=0$ and starting torque for the case with $w=1$ are inferior, as the respective function is omitted in the optimization process.

Table 1 Comparison of Performances by Individual Objectives ARPN IJEST

\begin{tabular}{|c|c|c|c|c|}
\hline$w$ & Motor & Performance & GA [11] & PM \\
\hline \multirow{4}{*}{1} & \multirow{2}{*}{1} & Eff & 86.708 & $\mathbf{8 6 . 7 4 2}$ \\
\cline { 3 - 5 } & & $T_{s t}$ & 0.857 & 0.755 \\
\cline { 2 - 5 } & \multirow{2}{*}{2} & $E f f$ & 90.497 & $\mathbf{9 0 . 5 7 4}$ \\
\cline { 3 - 5 } & & $T_{s t}$ & 0.395 & 0.368 \\
\hline \multirow{4}{*}{$\mathbf{0}$} & \multirow{2}{*}{1} & $E f f$ & 81.124 & 79.868 \\
\cline { 3 - 5 } & \multirow{2}{*}{2} & $T_{s t}$ & 10.063 & $\mathbf{1 0 . 3 5 4}$ \\
& & Eff & 84.139 & 84.340 \\
& & $T_{s t}$ & 16.142 & $\mathbf{1 6 . 4 4 8}$ \\
\hline
\end{tabular}

Table 2 Comparison of Results with multiple objectives for Motor-1

\begin{tabular}{|c|c|l|l|}
\cline { 3 - 4 } \multicolumn{1}{c|}{} & \multicolumn{1}{c|}{ GA } & \multicolumn{1}{|c|}{ PM } \\
\hline \multirow{4}{*}{ Primary } & $x_{1}$ & 1.91436 & 1.44334 \\
Design & $x_{2}$ & 0.44522 & 0.48141 \\
Variables & $x_{3}$ & 13311.74 & 12727.18 \\
$x$ & $x_{4}$ & 0.45166 & 0.44537 \\
& $x_{5}$ & 3.63006 & 3.95638 \\
& $x_{6}$ & 4.91137 & 4.72236 \\
\hline \multirow{2}{*}{ Constraints } & $x_{7}$ & 1.20089 & 1.20093 \\
$g(x)$ & $g_{1} \leq 2$ & 1.708 & 1.707 \\
& $g_{2} \leq 2$ & 1.097 & 1.154 \\
& $g_{3} \leq 0.05$ & 0.027 & 0.027 \\
\cline { 3 - 4 } & $g_{4} \geq 1.5$ & 12.498 & 12.402 \\
\hline
\end{tabular}

\begin{tabular}{|c|c|l|l|}
\hline \multirow{1}{*}{} & $g_{5} \leq 70$ & 33.778 & 37.180 \\
$g_{6} \leq 0.5$ & 0.498 & 0.500 \\
& $g_{7} \geq 0.75$ & 0.871 & 0.870 \\
\hline \multirow{2}{*}{ Performances } & $E f f$ & 84.559 & 84.608 \\
\cline { 2 - 4 } & $T_{s t}$ & 4.632 & 4.597 \\
\hline
\end{tabular}

The optimal design with multiple objectives are presented in Table 2 and 3 for motor 1 and 2 respectively. The corresponding performances in terms of efficiency and starting torque are also presented in the respective tables of 2 and 3. It can be observed from these tables that GA and HSO offer a compromised solution that lies in between the respective best and worst objective function values obtained with individual objectives. The quality of the compromised solutions cannot be estimated as it depends on the weight values assigned to the individual objectives and the range of the each objective function values. It is known that another compromised solution can be obtained by simply changing the weight parameter of each objective.

The Tables 2 and 3 also include the values of the constraints of Eq. (6) along with their limits. It can also be observed from these tables that both the methods bring the constraints such as maximum flux density, slip at full load, starting to full load torque ratio, etc., of Eq. (6) to lie within the respective limit, as the constraints are added as penalty terms in the fitness function of Eq. (14).

Table 3 Comparison of Results with multiple objectives for Motor-2

\begin{tabular}{|c|c|l|l|}
\cline { 3 - 4 } \multicolumn{1}{c|}{} & \multicolumn{1}{c|}{ GA } & \multicolumn{1}{c|}{ PM } \\
\hline \multirow{4}{*}{$\begin{array}{c}\text { Primary } \\
\text { Design }\end{array}$} & $x_{1}$ & 1.25331 & 1.98324 \\
Variables & $x_{2}$ & 0.39837 & 0.53788 \\
$x$ & $x_{4}$ & 11506.52 & 14699.59 \\
& $x_{5}$ & 0.89624 & 0.27214 \\
& $x_{6}$ & 3.62740 & 4.60577 \\
& $x_{7}$ & 1.10138 & 1.10044 \\
\hline \multirow{4}{*}{ Constraints } & $g_{1} \leq 2$ & 1.354 & 1.901 \\
$g(x)$ & $g_{2} \leq 2$ & 0.986 & 1.391 \\
& $g_{3} \leq 0.05$ & 0.018 & 0.016 \\
& $g_{4} \geq 1.5$ & 13.289 & 14.178 \\
& $g_{5} \leq 70$ & 25.807 & 37.390 \\
& $g_{6} \leq 0.5$ & 0.498 & 0.489 \\
& $g_{7} \geq 0.75$ & 0.850 & 0.855 \\
\hline \multirow{2}{*}{ Performances } & $E f f$ & 88.710 & 88.669 \\
\cline { 3 - 4 } & $T_{s t}$ & 4.710 & 5.295 \\
\hline
\end{tabular}

\section{CONCLUSIONS}

HSO is a powerful population based heuristic algorithm for solving multimodal optimization problems. An elegant methodology involving HSO for solving ODIM problem has been outlined. It determines the optimal values for primary design variables. The results on two IMs clearly demonstrates the ability of the PM to produce the global best design parameters that maximizes the efficiency and starting torque as well of the IM. It has been exhibited that the new approach 
encourages the continued use of HSO and will go a long way in serving as a useful tool in design problems.

\section{ACKNOWLEDGMENTS}

The authors gratefully acknowledge the authorities of Annamalai University for the facilities offered to carry out this work.

\section{REFERENCES}

[1] Kentli. K. (2009). A survey on design optimization studies of induction motors during the last decade, Journal of Electrical and Electronics Engineering, 9(2): 969-975.

[2] Menzies. R.W and Neal. G.W. (1975). Optimization program for large induction motor design, Proc.I.E.E., 11(6), 643-646.

[3] Gyeorye Lee, Seungjae Min, and Jung-Pyo Hong. (2013). Optimal shape design of rotor slot in squirrelcage induction motor considering torque characteristics, IEEE Transactions on Magnetics, 49(5): 2197-2200.

[4] Bharadwaj. D.G., Venkatesan.K and Saxena.R.B. (1978). Computer aided design of polyphase cage induction motors, Proc. Int. Conf. On Electrical Machines, Brussels, Belgium, 1(SP2/1): 1-10

[5] Faiz. J and Sharifian. M.B.B. (2001). Optimal design of three phase induction motors and their comparison with a typical industrial motor, Comp. and Elect. Eng. 27: 133144.

[6] Bharadwaj. D.G., Venkatesan.K and Saxena.R.B. (1979a). Induction motor design optimization using constrained Rosenbrock method (Hill Algorithm), Comput. Elec. Engg. 6(1): 41-46.

[7] Ramarathnam, R., Desai.B.G., and Subba Rao. V. (1973). A comparative study of minimization techniques for optimization of induction motor design. IEEE Transactions on Power Apparatus and Systems PAS-92 (5): 1448-1454.

[8] Parkin T. S and Preston T.W, (1993). Induction Motor Analysis Using Finite Element", Proc.IEE, The Eighth International Conference on Electrical Machines and Drives, 20-24.

[9] Bharadwaj. D.G, Venkatesan.K.and Saxena.R.B. (1979b). Nonlinear programming approach for optimum cost induction motors--SUMT algorithm, Comput. and Elect. Engg., 6(3): 199-204.

[10] Bhuvaneswari.R and Subramanian.S. (2005). Optimization of three phase induction motor design using simulated annealing algorithm, Electric Power Components and Systems, 33: 947-956.
[11] Prakash.P.S and Aravindhababu.P. (2014). GA based design for improving starting torque of induction motor, International Journal of Engineering Science and Technology, 6(12): 816-821.

[12] Jan Pawel Wieczorek, Ozdemir Gol and Zbigniew Michalewiez. (1998). An evolutionary algorithm for the optimal design of induction motors, IEEE Trans. Magnetic, 34(6): 3882-3887.

[13] Kim MK, Lee CG, Jung HK. (1998). Multiobjective optimal design of three-phase induction motor using improved evolution strategy, IEEE Trans. on Magnetics, 34(5): 2980-2983.

[14] Sakthivel. V.P, Bhuvaneswari. R and Subramanian. S. (2010-a). Economic design of three-phase induction motor by particle swarm optimization, J. Electromagnetic Analysis and Applications, 2: 301-310.

[15] Sakthivel. V.P, Bhuvaneswari. R and Subramanian. S. (2010-b). Design optimization of three-phase energy efficient induction motor using adaptive bacterial foraging algorithm, The International Journal for Computation and Mathematics in Electrical and Electronic Engineering, 29 (3): 699-726

[16] Thanga Raj.C, Radha Thangaraj, Millie Pant, Pascal Bouvry, and Ajith Abraham. (2012). Design optimization of induction motors with differential evolution algorithms with an application in textile spinning, Applied Artificial Intelligence, 26: 809-83.

[17] Geem. Z.W, Kim. J.-.H, Loganathan. G.V. (2001). A new heuristic optimization algorithm: harmony search, Simulation, 76(2): 60-68.

[18] Lee. K.S and Geem.Z.W. (2005). A new meta-heuristic algorithm for continuous engineering optimization: harmony search theory and practice, Comput. Methods Appl. Mech. Engrg. 194(3): 3902-3933.

[19] Prakash.P.S and Aravindhababu.P. (2014). Harmony search optimization based design of cost effective induction motor, International Review on Modelling and Simulations, 7(6): 912-917.

[20] Kumaran Jayaraman and Ravi G. (2014). Long-Term Forecasting of Electrical Energy Using ANN and HSA, International Review on Modelling and Simulations (IREMOS), 7(3): 489-496.

[21] Karthikaikannan Dharmaraj and Ravi G. (2014). Optimal Reactive Power Dispatch of Power System using Improved Harmony Search Algorithm, International Review on Modelling and Simulations (IREMOS), 9(3): 620-639. 